\author{
SAND93-8233 \\ Unlimited Release \\ Printed May, 1993
}

\title{
A Numerical Study of Hybrid Optimization Methods for the Molecular Conformation Problem
}

\author{
J. C. Meza and M.L. Martinez \\ Scientific Computing Department \\ Sandia National Laboratories \\ Livermore, CA 94551-0969
}

\begin{abstract}
An important area of research in computational biochemistry is the design of molecules for specific applications. The design of these molecules depends on the accurate determination of their three-dimensional structure or conformation. Under the assumption that molecules will settle into a configuration for which their energy is at a minimum, this design problem can be formulated as a global optimization problem. The solution of the molecular conformation problem can then be obtained, at least in principle, through any number of optimization algorithms. Unfortunately, it can easily be shown that there exist a large number of local minima for most molecules which makes this an extremely difficult problern for any standard optimization method.

In this study, we present results for various optimization algorithms applied to a molecular conformation problem. We include results for genetic algorithms, simulated annealing, direct search methods, and several gradient methods. The major result of this study is that none of these standard methods can be used in isolation to efficiently generate minimum energy configurations. We propose instead several hybrid methods that combine properties of several local optimization algorithms. These hybrid methods have yielded better results on representative test problems than single methods.
\end{abstract}

$3 / 4$ 


\section{Contents}

$\begin{array}{ll}\text { 1. Introduction } & 7\end{array}$

2. Numerical Methods $\quad 8$

2.1 Stochastic Methods . . . . . . . . . . . . . . . . . . 11

2.2 Direct Search Methods . . . . . . . . . . . . . . . . . . . 11

2.3 Gradient Methods . . . . . . . . . . . . . . . . . . 12

2.4 Hybrid Methods . . . . . . . . . . . . . . . . . . . 12

3. Numerical Results 12

3.1 Random Search . . . . . . . . . . . . . . . . . . . . 14

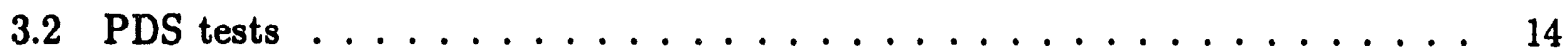

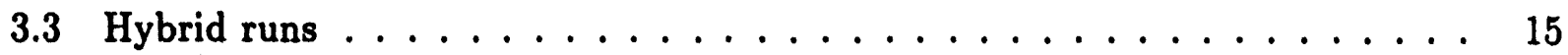

3.4 Distribution of Minima . . . . . . . . . . . . . . . . 17

3.5 Local Minimization Using Gradient Methods . . . . . . . . . . . . . . . . 19

4. Conclusions 19 


\section{Introduction}

An important area of research in computational biochemistry is the design of molecules for specific applications. Examples of these types of applications occur in the development of enzymes for the removal of toxic wastes, the development of new catalysts for material processing, and the design of new anti-cancer agents. The design of these drugs depends on the accurate determination of the structure of biological macro-molecules. This problem, known as the molecular conformation problem, consists of finding the configuration of a molecule that yields its lowest free energy. Although a molecule's linear or primary sequence is usually known, its three-dimensional structure, or conformation, usually is not known. Since the molecule's conformation determines whether a particular compound will perform its desired function, the determination of this structure is crucial to deciding whether any new compounds are useful. Under the assumption that the native structure of a molecule corresponds to a conformation for which the energy is at or near the global minimum, the molecular conformation problem can be formulated as a global optimization problem.

One approach to the molecular conformation problem then is to formulate it as a constrained global minimization problem which can be solved using standard numerical techniques. Unfortunately, since the total energy of a molecule depends on atom-atom interactions, the number of possible low-energy configurations can grow exponentially with the number of atoms and has been estimated by Hoare to be on the order of $O\left(e^{N^{2}}\right)$ for an $N$-atom molecule [8]. The large number of local minima that the energy functions possess makes it an extremely difficult problem for all standard optimization methods. It is also important to note that although the problem is formulated as a global optimization problem, nearby low-energy configurations are also of interest to biochemists. These nearby minima are of interest because they correspond to alternate states that the molecule can occupy.

The purpose of this study is to consider the use of hybrid optimization algorithms that use a combination of several methods in different regions of the parameter space. In an earlier study [9] it was found that a single optimization algorithm was often not as effective as a combination of optimization methods. Other recent attempts at solving the molecular configuration problem using parallel stochastic methods as in [2,3], and direct optimization methods as in $[12,17]$, suggest that these alternatives to single optimization methods are more successful. In this study, we pursue the approach of using hybrid methods composed of two or more methods that operate in different regions of the search strategy. We will decompose the search for a glo -1 minimum into two distinct phases: a global search phase and a local minimization phase. In the global search phase or global phase, we are interested in generating a distribution of good initial guesses for the second phase. In the local phase we assume that the initial guess provided by the global phase is inside a region of attraction for some local minima that is close to the global minimum.

In Section 2 we describe the optimization methods used in this numerical study and motivate the need for the hybrid approach. Section 3 contains the numerical results obtained from our test problems. We conclude in Section 4 with a discussion on the performance of these hybrid methods and some conclusions. 


\section{Numerical Methods}

Before introducing the various optimization methods used, we will describe the model problem used and some of its characteristics. As in [9], we used a two-dimensional polymer consisting of $N$ atoms connected by rigid rods of unit length. The function used to describe the energy of this system is given by a pairwise additive function consisting of Lennard-Jones potentials, that is,

$$
V(\Theta[r])=\sum_{i, j>i}^{N}\left[\left(\frac{\alpha}{r_{i j}}\right)^{12}-2\left(\frac{\alpha}{r_{i j}}\right)^{6}\right],
$$

where $r_{i j}$ is the distance between the atoms $x_{i}$ and $x_{j}, \Theta$ is the bond angle between any three consecutive atoms, and $\alpha$ is a constant. If we set $\alpha=1$, the minimum for a given pair of atoms will correspond to a unit distance, and the global minimum corresponds to closed hexagonal packs with unit spacing.

There are two approaches for finding a minimum to 1. The first approach involves minimization with respect to the spatial coordinates of the atoms and enforcing the bonclength constraints explicitly. The second approach, which we have undertaken, is to convert the entire system to a set of internal coordinates described by the bond angles between adjacent pairs of atoms. In this way, the nonlinear constraints are easily enforced at the expense of a coordinate transformation each time a function evaluation is needed. This also has the advantage of allowing us to use unconstrained optimization algorithms.

One of the interesting results of the earlier study [9] is that the parameter space can be visualized as having two easily distinguished regions. The first region is characterized by high energies and corresponds to configurations that are knotted. The second region is characterized by low energies and corresponds to un-knotted configurations. The probability that a random configuration will have at least one knot (and therefore fall into the first region) is a function of the number of atoms in a molecule with that probability approaching one for even moderately sized molecules. For a molecule with 61 atoms, for example, the probability is already greater than $99.9999 \%$ that it will have at least one knot. Additionally, the energies for two configurations in the two regions can differ by 15-16 orders of magnitude. Figures 12 show two possible configurations that are representative of the two energy regions.

These points are worth noting because the previous numerical studies showed that it was difficult for any single optimization algorithm to start at a random high-energy configuration and reach a good approximation to the global minimum. Those algorithms that could make the transition from the high-energy region to the low-energy region exhibited slow progress towards a minimum in the low energy region. Alternately, the algorithms that are known to have good local convergence rates did not often reach the low-energy region from a random starting point. As such, we were led to investigate the properties of hybrid methods that could incorporate the best properties of each type of method.

For the purposes of this paper, we will categorize optimization methods as either stochastic or deterministic. Stochastic methods incorporate a random element in their search strategies. Examples of stochastic methods are random search, genetic algorithms and simulated annealing. Deterministic methods are those that do not incorporate a random element into 


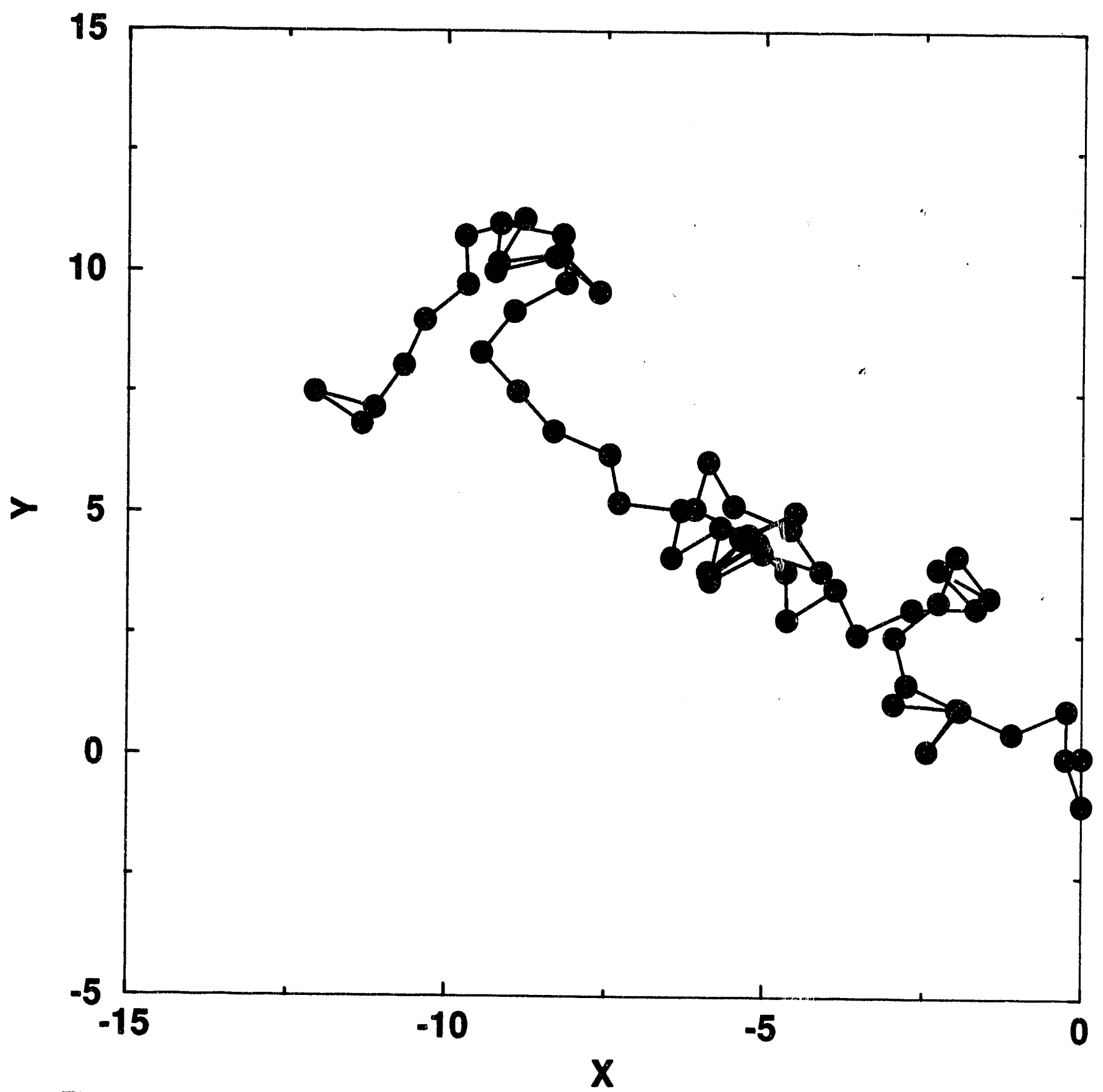

Figure 1: Knotted high-energy configuration corresponding to a 61 atom molecule with an approximate energy of $10^{15}$. 


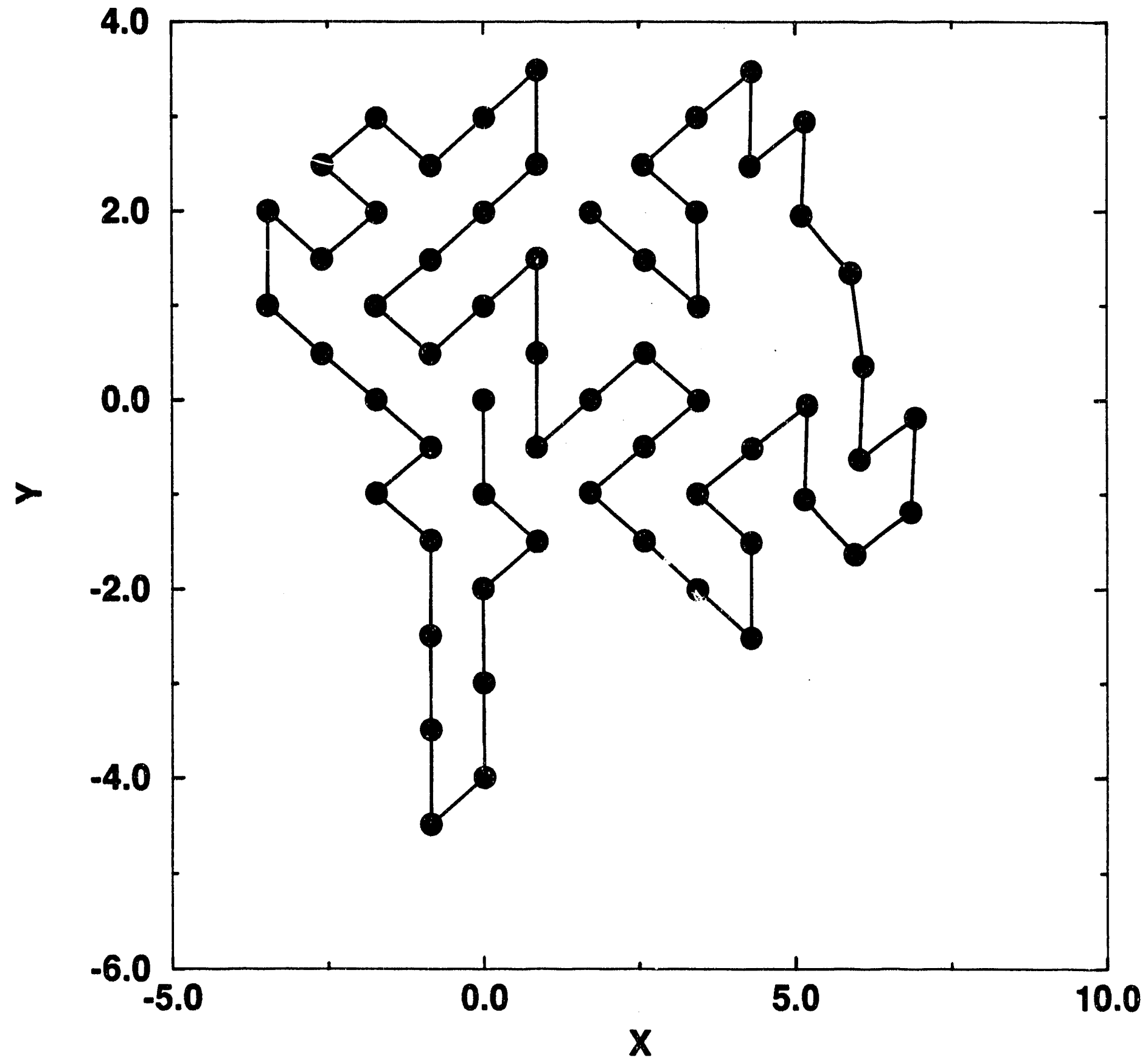

Figure 2: Un-knotted low-energy configuration corresponding to a 61 atom molecule with an approximate energy of -100 . 
their behavior. Examples of deterministic methods we studied are direct search methods, conjugate gradient methods, and Newton methods. The rest of this section is devoted to briefly describing these methods.

\subsection{Stochastic Methods}

Perhaps the earliest attempts at finding global minima used a technique known as random search. In its simplest form this method generates random initial guesses, compares function valucs, and stores the lowest function value found. A slightly more sophisticated version of this method adds a local minimization step from each random initial guess and keeps the lowest function value found after the minimization phase.

Genetic algorithms $(\mathrm{GA})$ [7] are optimization methods based on analogies to natural selection strategies from evolution. The idea is to encode the problem parameters as a binary bit string called a gene. A population of these binary genes are randomly generated and then ranked according to a measure of fitness which in most cases is taken to be the value of the objective function. A new generation of genes is then created using two concepts called crossover and mutation. In crossover, two parent genes are chosen and two new genes are produced by combining a subset of the genes from one parent gene with a subset from the other parent. Mutation represents the changing of a single bit of a binary gene.

Simulated annealing [10] (SA) is another stochastic optimization method and is based on a thermodynamic process called annealing. It is known that whenever a liquid is allowed to cool slowly, it will settle into its lowest-energy state or global minimurn. Interestingly, if the cooling process is done rapidly the liquid will not settle into its lowest energy state but instead settle into a local minimum. In the case of metals, this process is known as annealing. This physical process can be mimicked in an optimization algorithm by allowing the algorithm to accept steps that not only decrease the function value, but with some probability will also accept steps that increase the function value. The probability of accepting a step that increases the objective function value depends on another function of a parameter known as the temperature. This function is designed such that for high values of the temperature the probability of accepting a step that increases the objective function value is large and for low temperatures the probability is small. In simulated annealing, this temperature parameter is initially set high so that a large proportion of uphill directions are accepted and is slowly decreased in an attempt to simulate the annealing process.

Both GA and SA have the property that they allow uphill directions and the iterates will therefore not necessarily decrease monotonically. This property is sometimes used to justify the assertion that GA and SA are global optimization methods because they can jump out of local wells by moving uphill. In the case of simulated annealing, Dekkers and Aarts [4] have proven asymptotic convergence to a set of global minimum. We are unaware of any convergence proofs for genetic algorithms applied to global optimization problems.

\subsection{Direct Search Methods}

Direct search methods belong to a class of optimization methods that do not compute derivatives. Examples of direct search methods are the Nelder-Mead simplex method, pattern 
search, and the box method. Dennis and Torczon's parallel direct search (PDS) algorithm is an extension of the Nelder-Mead simplex method that uses the concept of searching in several directions simultaneously. Starting from an initial simplex, $S_{0}$, the function value at each of the vertices of $S_{0}$ is computed and the vertex corresponding to the lowest function value, $v_{0}$, is determined. The simplex $S_{0}$ is rotated $180^{\circ}$ about $v_{0}$ and the function values at the vertices of this rotation simplex, $S_{r}^{\prime}$, are compared against $v_{0}$. If one of the vertices in $S_{r}$ has a function value lower than $v_{0}$, then the simplex, $S_{r}$, is expanded to form the simplex $S_{e}$. The function values at the vertices of $S_{e}$ are compared against the lowest function value found in $S_{r}$. If a lower function value is encountered, then $S_{e}$ is accepted as the starting simplex for the next iteration, otherwise $S_{r}$ is accepted for the next iteration. If at the time that $S_{r}$ is constructed no function value lower than $v_{0}$ is found in $S_{r}$, then a contraction simplex, $S_{c}$ is created by reducing the size of $S_{0}$ by some multiple, and the contracted simplex is accepted as the new simplex for the next iteration.

Because PDS only uses function comparisons it is relatively easy to implement and use. Since the rotation, expansion, and contraction steps are all well-determined it is also possible to determine ahead of time a set of grid points corresponding to the vertices of simplices constructed from various combinations of rotations, expansions, and contractions. Given this set of grid points, called a search scheme, the PDS algorithm can compute the function values at all of these vertices in parallel and take the vertex corresponding to the lowest function value. One approach therefore is to take the scheme size to be equal to the number of processors available. Another approach, which we have sollowed, is to vary the size of the search scheme in an attempt to sample the parameter space for the global minimum. Used in this manner, PDS has the property that it can jump over barriers in what would amount to uphill directions. The PDS algorithm can also be shown to converge under some mild assumptions [15].

\subsection{Gradient Methods}

In the case that derivatives are available analytically or can be easily computed, it is useful to take advantage of this higher order information. Two examples of these types of methods include conjugate gradient (CG) methods and Newton methods. In our study, we used several versions of each type of method. In [9] a conjugate gradient method from [16] was used. For the purposes of direct comparison, in this study we also performed some tests using this implementation of the conjugate gradient method. We also used a limited memory BFGS method developed by Nocedal, and a quasi-Newton method with a BFGS update formula developed by Gay. The last two methods in particular possess good local convergence properties. Details of these methods can be found in $[6,11]$.

\subsection{Hybrid Methods}

Early on in our numerical studies we found that the use of a single optimization mf inod was usually not effective in reaching a minimum except in the case of a small dimensional problem. This observation led us to investigate the properties of hybrid algorithms that combined two or more of the standard optimization methods described above. Specifically, 
by a hybrid method for global optimization we will mean any algorithm that consists of a combination of optimization algorithms that can be separated into a global phase and a local phase. The goal of the global phase method is to search the parameter space for points that can be used as starting points for the local phase. The local method then refines the search started by the global phase method.

Using this definition, random search can be viewed as a primitive hybrid method where the global phase consists of randomly picking points and the local phase is either direct function comparisons or some local minimization procedure. In [9], Simulated Annealing and Genetic Algorithms were used in the global phase in conjunction with a CG method for the local phiase. This numerical study extends that work and consists of hybrid methods that use the PDS algorithm for the global phase. Specifically we compare the performance of PDS as a global phase method against stochastic methods such as Simulated Annealing (SA) and Genetic Algorithms (GA).

\section{Numerical Results}

In [9] it was noted that a combination of algorithms yielded better results than a single method. In this study, we explore these ideas in more detail. As such, the tests include several optimization algorithms described in the previous section, as well as combinations of these algorithms. The PDS code used is a slight modification to a code developed by Torczon and obtained from the Center for Research in Parallel Computations at Rice University. The CG code used was taken from [16]. The limited memory BFGS (LBFGS) method was provided by Nocedal [11]. The quasi-Newton method, SUMSL, was developed by D. Gay. All numerical tests were run on SGI workstations using IEEE double precision arithmetic with a machine precision, $\mu \approx 1.1 \cdot 10^{-16}$.

Using the model problem (1) we tested various combinations of methods for molecules with different numbers of atoms. We studied molecules of 19,37 , and 61 atoms because the minima correspond to configurations that are hexagonal closed packs of radii 2,3 , and 4 . Each test case consisted of running a set of trials starting from a set of randomly chosen starting points. To provide consistency across all of the test cases a set of 1000 configurations was generated from a uniform random distribution and stored in a file. This file was then used as the set of initial guesses for all of the test cases.

Because of the different methods used there were three different stopping criteria used. The PDS method uses a step tolerance, that is, the method terminates whenever the following condition is met:

$$
\frac{1}{\Delta}\left(\max _{i, j}\left\|v_{i}-v_{j}\right\|_{2}\right) \leq \mathrm{XTOL},
$$

where $\Delta=\max \left(1,\left\|v_{0}\right\|_{2}\right)$, and $v_{0}$ is the initial guess. The conjugate gradient method from [16] used a convergence criteria based on the function values:

$$
2 \frac{\left|f_{k+1}-f_{k}\right|}{\left|f_{k+1}\right|+\left|f_{k}\right|+\epsilon} \leq \text { FTOL, }
$$

where $\epsilon$ is equal to the machine precision. For the LBFGS method, the convergence test 
Table 1: Minimum energies found using random search for 61 atom case.

\begin{tabular}{lr}
\hline Method & Minimum \\
\hline RANDOM & 5540000.0 \\
CG & 38402.0 \\
LBFGS & 1328.4 \\
SUMSL & 628.8 \\
PDS $(2360)$ & -117.5 \\
\hline Exact & -170.5 \\
\hline
\end{tabular}

used consists of a test on the gradient, that is,

$$
\left\|g_{k}\right\| \leq \text { GTOL }\left(\max \left(1,\left\|x_{k}\right\|\right)\right) .
$$

Unless otherwise indicated, all of our tests used the following tolerances: XTOL $=10^{-3}$, FTOL $=10^{-5}$, and GTOL $=10^{-5}$.

\subsection{Random Search}

The first set of test problems was devised to test the hypothesis that a single optimization method would not be effective. Table 1 shows the results using a single optimization method for the case of 61 atoms. The number of trials in each case is 100, except for the first entry labeled RANDOM. Since this case merely reads an initial guess from a file and computes its function value each trial is relatively inexpensive. In this case only, the number of trials was set to 100,000 . In the case of PDS, the search scheme size was set to 2360 points, denoted by PDS(2360), although the results are similar for various sizes of the search scheme.

It is worth noting that the only method to get a negative energy was PDS. Although this energy is still far from the global minimum of -170.5 , any negative energy corresponds to a configuration without knots which in turn corresponds more closely to structures known to be close to the global minimum.

\subsection{PDS tests}

Since the only method that succeeded in generating a negative energy starting from a random initial guess was PDS, we proceeded to investigate this method in more detail. One question that arises immediately is whether the size of the search scheme has an effect on the minimum found. Clearly the larger the scheme size the larger the sampling of the parameter space. The disadvantage is that each iteration will be more costly. In the next set of test cases, we chose the simplex shape to be regular, allowing PDS to construct the entire initial simplex from the initial random configuration. We considered search scheme sizes (SSS) of $2 m, 5 m, 10 m, 20 m$, and $40 m$ where $m=N-2$ for a molecule of $N$-atoms and ran 100 trials for each test case. Table 2 contains the results for the 61 atom case. 
Table 2: Comparison of different PDS search size schemes (SSS) for 61 atom case.

\begin{tabular}{rrr}
\hline Method & Minimum & \% Negative \\
\hline PDS(118) & -92.63 & 0.5 \\
PDS(295) & -115.95 & 45.0 \\
PDS(590) & -113.94 & 78.0 \\
PDS(1180) & -115.02 & 83.0 \\
PDS $(2360)$ & -117.47 & 85.0 \\
\hline
\end{tabular}

As Table 2 shows, all of the test cases yielded configurations with negative energies. The third column of Table 2 also displays the percentage of all trials that yielded a negative energy. In a sense, this is an indication of how robust the method is. We note that with a search scheme size of $10 \mathrm{~m}$ approximately $80 \%$ of the trials had configurations with negative energies. As the search scheme size increases we get a higher percentage of trials with negative energies but at the cost of substantially more function evaluations per iteration. Figure 3 contains a representative configuration for the 61 atom test case computed by PDS using a search scheme size of 2360 points.

\subsection{Hybrid runs}

The previous study [9] indicated that both GA and SA yielded good approximations to the global minimum. The next step in our numerical experiments was to test the combination of PDS with other local methods. For the purposes of a direct comparison, we ran a test case using PDS with the same CG routine used in [9]. The lowest energies found are given in Table 3 and compared against the best known global minimum energy.

For the 19 and 37 atom test cases, the results are similar in terms of the final energies. In the 61-atom case, the various methods start to exhibit greater differences. In this case, the lowest energies computed by GA with CG and SA with CG are much closer to the best known global minimum than PDS with CG. Table 3 also indicates that PDS can be used in a similar manner to GA or SA, that is as the glcbal phase of a two-phase algorithm.

The next set of tests combine PDS with gradient methods. All of these tests use PDS with a value of SSS of $40 m=2360$. From the results using random search we can predict that it would not be useful to start a local minimization from a configuration that has a large energy value. We therefore modified our algorithm to set a user-defined energy tolerance such that if the value returned from the global phase (in this case PDS) is higher than this energy tolerance, the local phase is skipped.

We also note that PDS was modified for the local phase so that it would restart after it had converged using the best vertex as its initial guess for the restart. To allow for greater flexibility, we allowed the user to set the maximum number of restarts. In these tests we set the number of restarts equal to 5 . 


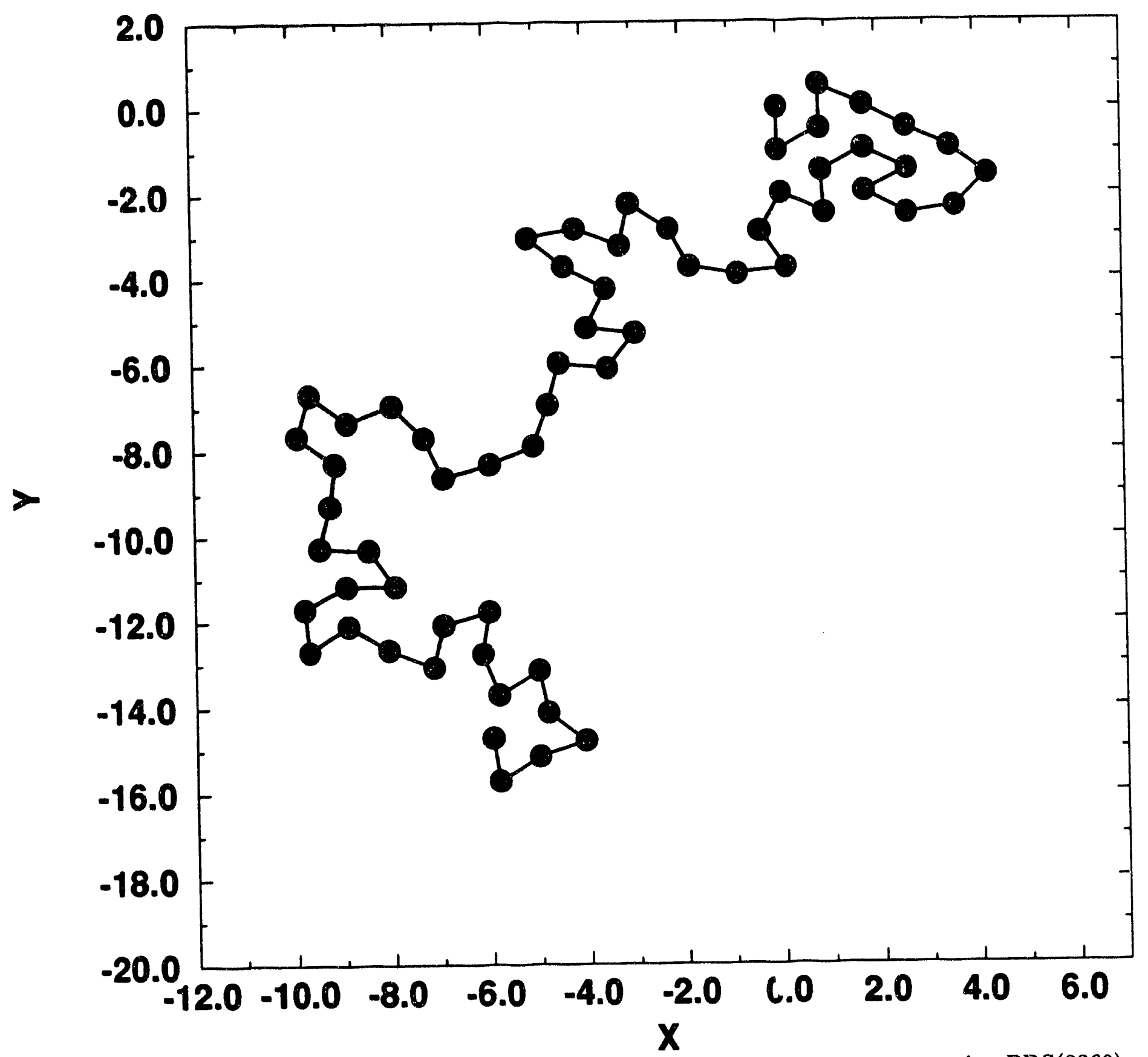

Figure 3: Mininum energy conformation computed for a 61 atom test case using PDS(2360). 
Table 3: Comparison of PDS versus GA and SA for 19, 37, and 61 atom test cases.

\begin{tabular}{cc|ccc}
\hline \multicolumn{2}{c|}{ Method } & \multicolumn{3}{c}{ Minimum } \\
\hline Global & Local & 19 atoms & 37 atoms & 61 atoms \\
\hline GA & CG & -44.3 & -97.3 & -166.6 \\
SA & CG & -44.2 & -94.8 & -164.4 \\
PDS & CG & -45.3 & -95.3 & -142.6 \\
\multicolumn{2}{c|}{ Best Known } & -45.3 & -98.3 & -170.5 \\
\hline
\end{tabular}

Table 4: Minimum energies found using PDS as the global phase method for 61 atom case.

\begin{tabular}{ccc}
\hline \multicolumn{2}{c}{ Hybrid Method } & \\
\cline { 1 - 2 } Global & Local & Energy \\
\hline PDS(2360) & PDS(2360) & -117.5 \\
PDS(2360) & CG & -142.6 \\
PDS(2360) & LBFGS & -167.2 \\
PDS(2360) & SMSNO & -166.0 \\
PDS(2360) & SUMSL & -163.6 \\
\hline
\end{tabular}

The results of these tests are displayed in Table 4. The first observation we make is that PDS is not as effective in the local phase as any of the gradient based methods. Even with restarts, PDS does not get a solution near a minimum. Theoretically PDS should not converge to a non-minimizer so this behavior seemed unusual at first. In fact, if the search scheme size is increased at the same time that a restart occurs, then PDS will start to progress towards a minimum again, although at an extremely slow rate. For the gradient based methods, the results are all similar in terms of the final energy. The combination of PDS+LBFGS yielded the lowest energy overall, but not substantially better than the other methods. The difference between SUMSL and SMSNO lies in the availability of first derivatives. The SMSNO method uses finite differences to compute gradients, whereas SUMSL uses ar:-jytic gradients. For these test problems, we used ADIFOR to generate analytic derivatives [1].

Figure 4 contains a representative configuration computed by a hybrid method using PDS(2360) for the global phase and SUMSL in the local phase.

\subsection{Distribution of Minima}

Although our main goal is to find the global minimum it is also important to find local minima that might be close to the global minimum. These configurations are of interest 


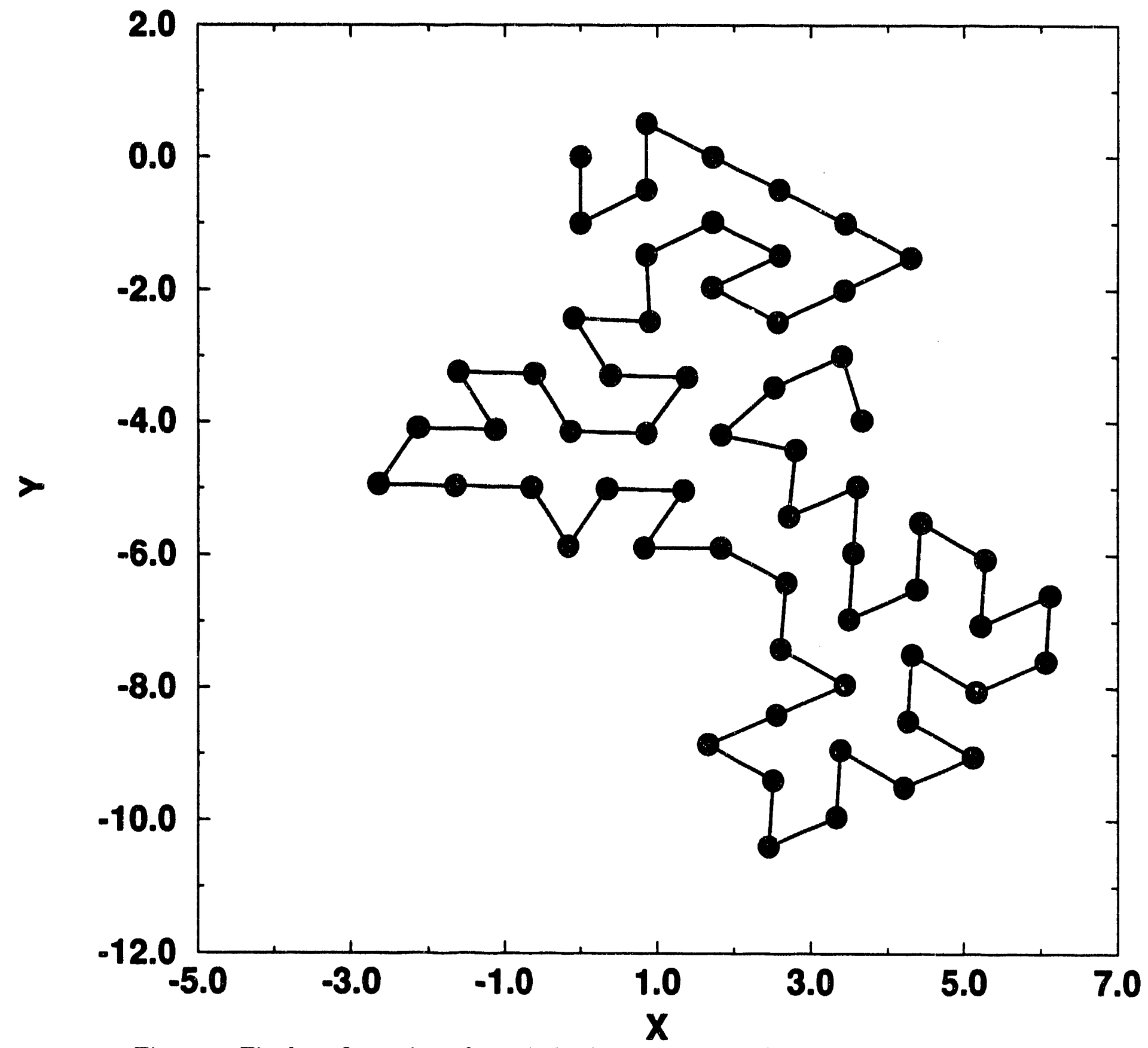

Figure 4: Final configuration after a hybrid run using PDS(2360) + SUMSL for $\mathrm{N}=61$ atom tist case. 
because they might correspond to natural variations of a given molecule that are of chemical interest. One way of depicting this result is to generate a distribution of the local minima found for each method. Figure 5 contains the distributions for the test cases corresponding to the 61 atom molecule. The three curves using PDS(2360) as the global phase all compare favorably with the distribution corresponding to the test results using $\mathrm{GA}$ as the global phase. In fact, all four curves generate distributions with at least $80 \%$ of the final configurations having negative energies. Simulated annealing (SA) is the only method that does not do well on this problem. We also note that of these methods, the combination of PDS(2360) with SUMSL yields the best distribution with approximately $80 \%$ of the minima having energies below -144.0.

\subsection{Local Minimization Using Gradient Methods}

Several interesting results from the gradient based minimization algorithrns are worth mentioning. The first point is that even with a good initial guess, for example a low-energy, un-knotted configuration, all of the gradient methods are slow to converge. In almost all situations the convergence criteria used to terminate the algorithm was based on the step size and not on the size of the gradient. Although both LBFGS and the quasi-Newton methods showed good initial progress, both methods slowed down exhibiting à slow linear rate of convergence. Figure 6 contains a typical iteration summary from a test case for a 61 atom test problem starting with the solution generated in the global phase by PDS(2360).

The three curves correspond to using LBFGS with analytic gradients, SUMSL (BFGS with analytic derivatives), and SMSNO (BFGS with finite-difference gradients). For this particular test case, SUMSL required 318 iterations to reduce the function value from -54.8 to -163.63 with a final gradient norm of 0.01039 . Judging from the norm of the gradient it appears that this point is far from a minimum, but the corresponding configuration could be considered good. It is also clear that the availability of analytic gradients does not have much of an effec' on the nonlinear iteration. An eigenvalue analysis of the Hessian at the solution showed that it is slightly ill-conditioned (with a condition number on the order of $10^{4}$ ), but certainly not significant when compared to machine precision which is on the order of $10^{-16}$. At this point, it is not clear why these methods are exhibiting this type of behavior and these issues will bear further study.

\section{Conclusions}

We can make several conclusions from the numerical results. The first point is that because of the two distinct regions of the parameter space a single optimization method will not be effective. The methods that did not use derivative information such as GA, SA, and PDS could all make the transition into the low energy region but once inside this region they exhibited a very slow convergence rate. The methods that used derivative information never succeeded in making it into the low energy region from any starting point outside the low energy region. If a random starting point happeneil to fall inside the low energy region the gradient based methods worked well, but as we pointed out in Section 2 the probability of this happening for even moderately sized molecules is miniscule. 


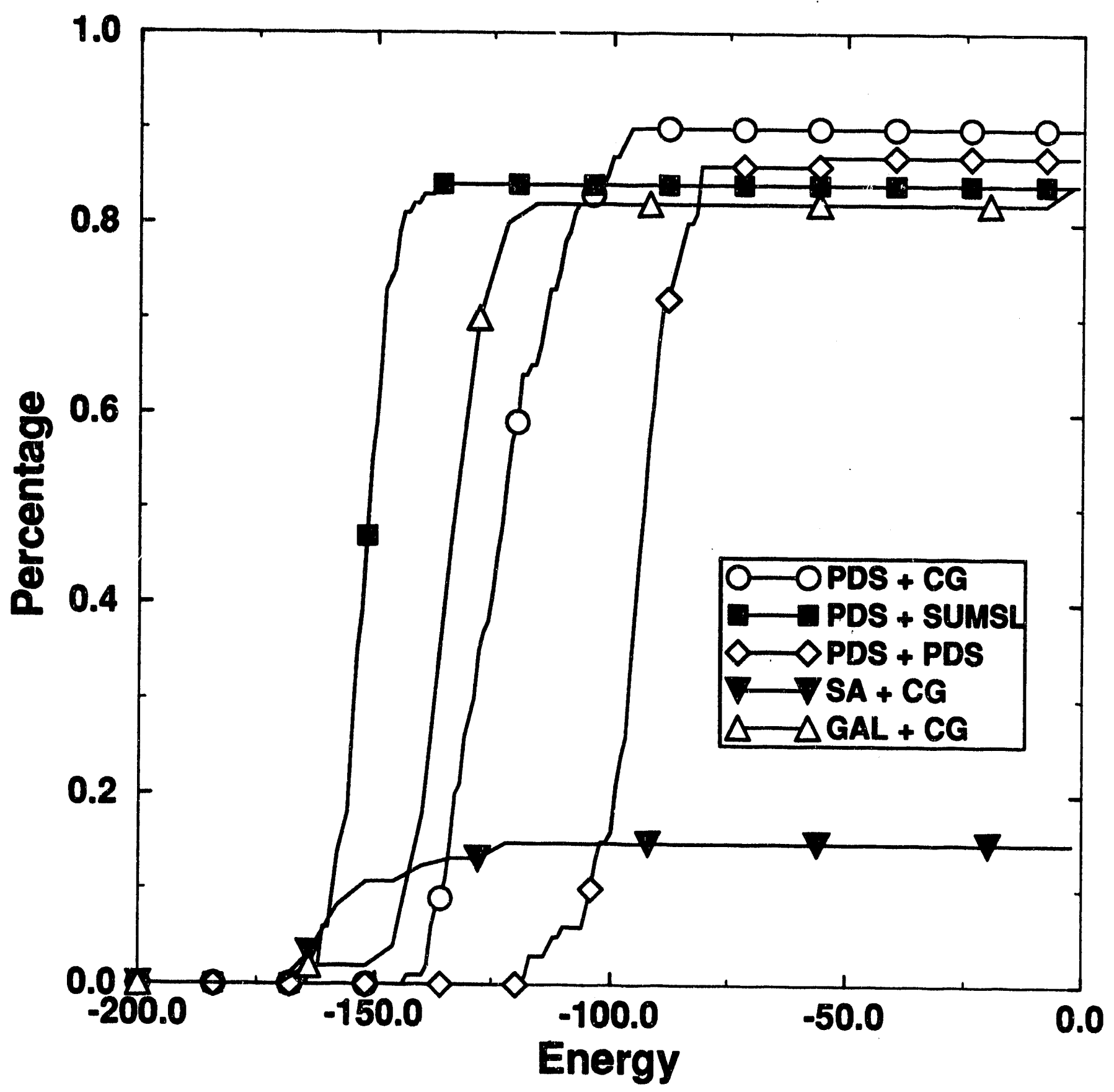

Figure 5: Distribution of minima for $\mathrm{N}=61$ atom test case. 


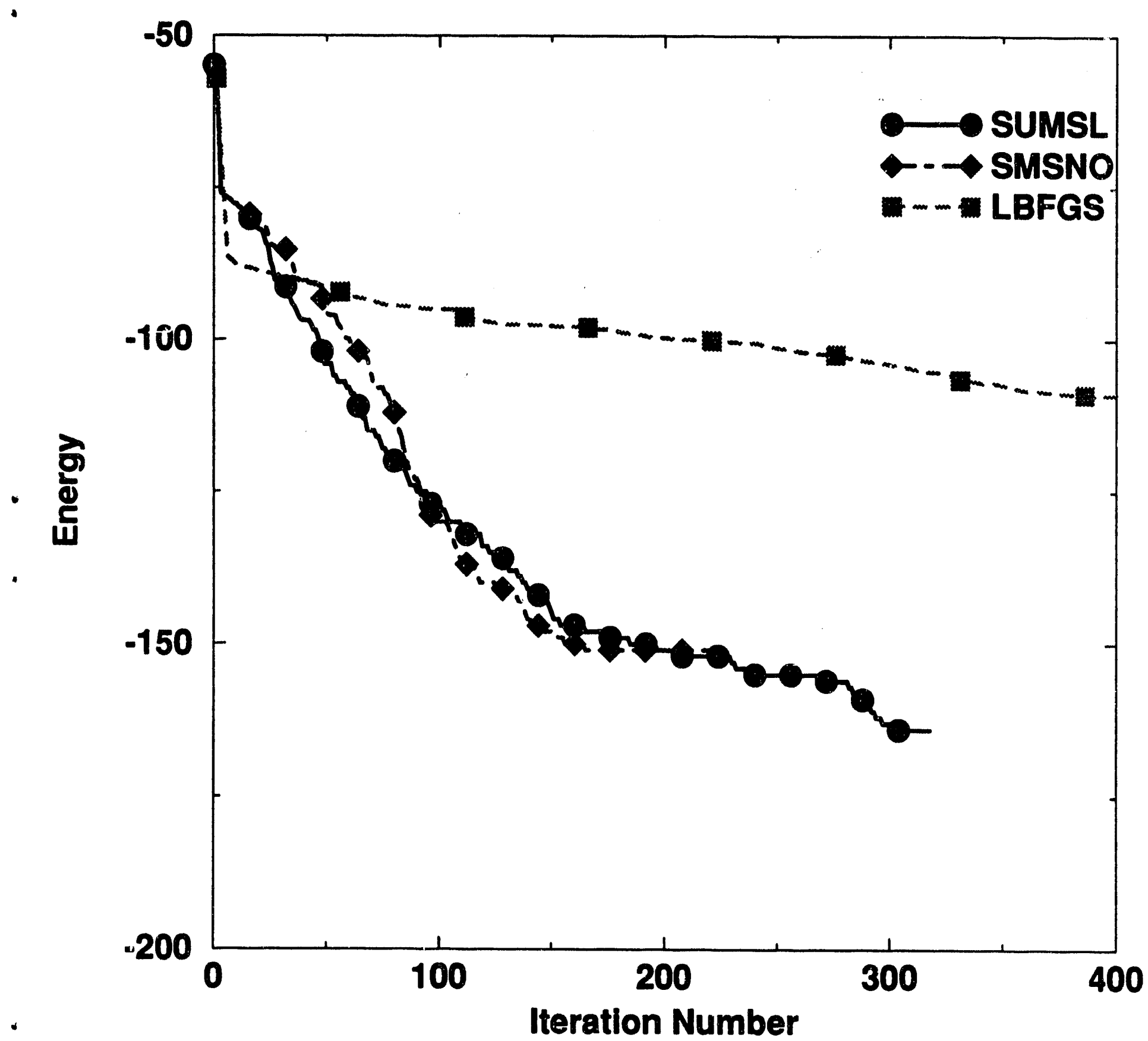

Figure 6: Iteration summary for three local optimization algorithms, $\mathrm{N}=61$ atom test case. 
In contrast, hybrid methods that incorporate the properties of several methods tend to work well. In [9], we studied GA and SA with the conclusion that GA served well as the first pass of a multi-pass algorithm. In this paper, we showed that PDS can also be used in a similar manner. When PDS is used in the global phase it can usually succeed in generating a point inside the low energy region. The effect of the search scheme size has some effect on the success ratio, but after a certain search scheme size is reached there are marginal returns. The PDS algorithm can also be shown to converge under some mild conditions.

A minor point that was discovered during the course of the numerical experiments is that there was no appreciable difference between using analytic gradients and finite difference approximations to the derivative in the quasi-Newton methods.

Further research will continue on several fronts. This problem is still a difficult problem once a configuration is found in the low energy region. A typical trial using a quasi-Newton method averages over 300 iterations to converge. Further work remains on improving this phase of the optimization. In addition, we will investigate the use of constrained optimization algorithms. As this problem is initially formulated it is a constrained optimization problem. Using a transformation we recast the problem into an unconstrained problem so that we could use algorithms that took advantage of this simpler formulation. Our next step will be to investigate the use of optimization techniques for the original constrained problem. 


\section{REFERENCES}

[1] C. Bischof, A. Carle, G. Corliss, A. Greiewank, and P. Hovland. ADIFOR-generating derivative codes from Fortan programs. Scientific Programming, 1(1):11-29, 1992.

[2] R.H. Byrd, E. Eskow, and R.B. Schnabel. A new large-scale global optimization method and its application to Lennard-Jones problems. Technical Report CU-CS-630-92, University of Colorado at Boulder, 1992.

[3] R.H Byrd, E. Eskow, R.B. Schnabel, and S.L. Smith. Parallel global optimization: Numericel methods, dynamic scheduling methods, and applications to molecular configuration. Technical Report CUCS-553-91, University of Colorado at Boulder, 1991.

[4] A. Dekkers and E. Aarts. Global optimization and simulated annealing. Mathematical Programming, 50:367-393, 1991.

[5] J.E. Dennis and R.B. Schnabel. Numerical Methods for Unconstrained Optimization and Nonlinear Equations. Prentice Hall, Englewood Cliffs, New Jersey, 1983.

[6] D.E. Goldberg. Genetic Algorithms in Search, Optimization, and Machine Learning. Addison-Wesley, 1989.

[7] M.R. Hoare. Structure and dynamics of simple microclusters. Adv. Chem. Phys., 40:49-135, 1979.

[8] R.S Judson, M.E. Colvin, J.C. Meza, A. Huffer, and D. Gutierrez. Do intelligent configuration search techniques outperform random search for large molecules? International Journal of Quantum Chemistry, 44:277-290, 1992.

[9] S. Kirkpatrick, C.C. Gelatt, and M.P Vecchi. Optimization by simulated annealing. Science, 220:671-$680,1983$.

[10] D.C. Liu and J. Nocedal. On the limited memory BFGS method for large scale optimization. Mathematical Programming, 45:503-528, 1989.

[11] R.S. Maier, J.B. Rosen, and G.L. Xue. Discrete-continuous algorithm for molecular energy minimization. Technical Report 92-031, AHPCRC, 1992.

[12] W.H. Press, B.P. Flannery, S.A. Teukolsky, and W.T. Vetterling. Numerical Recipes. Cambridge University Press, Cambridge, 1987.

[13] V.J. Torczon. On convergence of multi-directional search algorithm. SIAM J. Optimization, 1(1):123$145,1991$.

[14] G.L. Xue. Improvement on the Northby algorithm for molecular conformation: Better solutions. Technical Report 92-055, University of Minnesota, 1992. 


\section{UNLIMITED RELEASE}

INITIAL DISTRIBUTION:

Dr. Paul Boggs

National Institute of Standards and Technology

Building 101 A-238

Gaithersburg, MD 20899

Dr. Richard H. Byrd

University of Colorado

Department of Computer Science

Campus Box 430

Boulder, CO 80309

Dr. Maria Rosa Celis

Silicon Graphics Computers

M/S 9L/420

2011 N. Shoreline Dr.

Mountain View, CA 94039

Dr. Thomas F. Coleman

Cornell University

Department of Computer Science

Upson Hall

Ithaca, NY 14853-7501

Dr. Edward J. Dean

University of Houston

Department of Mathematics

4800 Calhoun Rd.

Houston, TX 77004-2610

Yuefan Deng

Applied Mathematics Department

SUNY at Stony Brook

Stony Brook, NY 11794-3600

Dr. John E. Dennis, Jr.

Department of Mathematical Sciences

Rice University

P.O. Box 1892

Houston, TX 77251-1892

Dr. James Glimm

Applied Mathematics and Statistics

P138A Mathematics

SUNY at Stony Brook

Stony Brook, NY 11794-3600 
Dr. Jorge More

Argonne National Laboratory

Mathematics and Computer

Science Division

Argonne, IL 60439-4803

Dr. Stephen Nash

Operations Research and Applied Statistics

George Mason University

Fairfax, VA 22030-4444

Dr. Jorge Nocedal

Department of Electrical Engineering and Computer Science

Northwestern University

Evanstown, IL 60208-0001

Dr. J. Ben Rosen

University of Minnesota

Department of Computer Science

200 Union Street SE

Minneapolis, MN 55455-0154

Dr. Robert Schnabol

Department of Computer Science

University of Colorado at Boulder

Campus Box 430

Boulder, Colorado 80309-0430

Dr. William Symes

Depariment of Mathematical Sciences

Rice University

P.O. Box 1892

Houston, TX 77251-1892

Dr. Richard Tapia

Department of Mathematical Sciences

Rice University

P.O. Box 1892

Houston, TX 77251-1892

Dr. Virginia Torczon

Department of Mathematical Sciences

Rice University

P.O. Box 1892

Houston, TX 77251-1892 


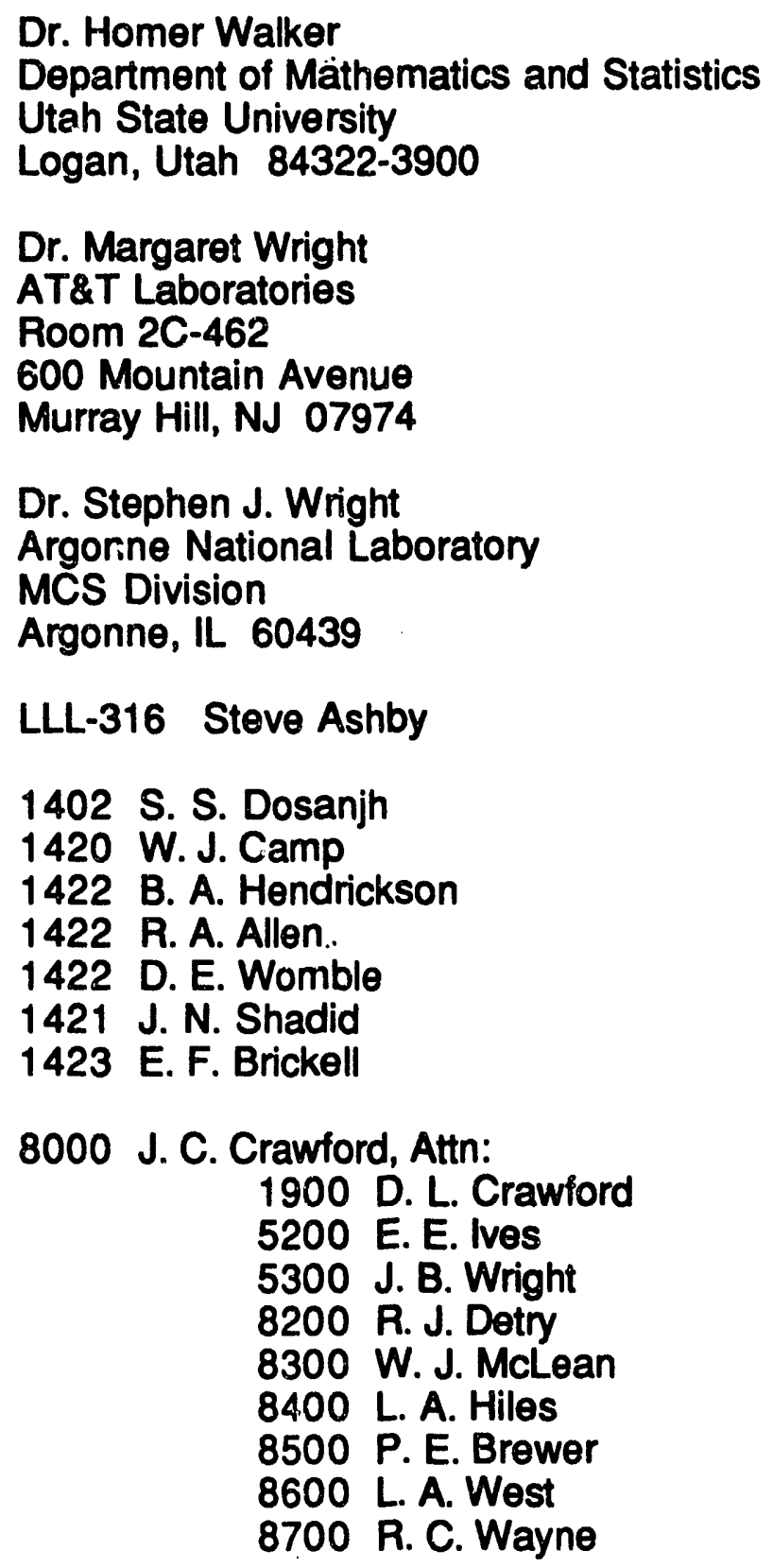

8100 M. E. John

8112 L. D. Brandt

8114 R. J. Gallagher

8117 M. E. Colvin

8117 R. S. Judson

8117 J. C. Meza'(20)

8535 Publications for OSTI (2)

8535 Publications/Technical Library Processing, 7141

7141 Technical Library Processes (3)

8524-2 Central Technical Files (3) 

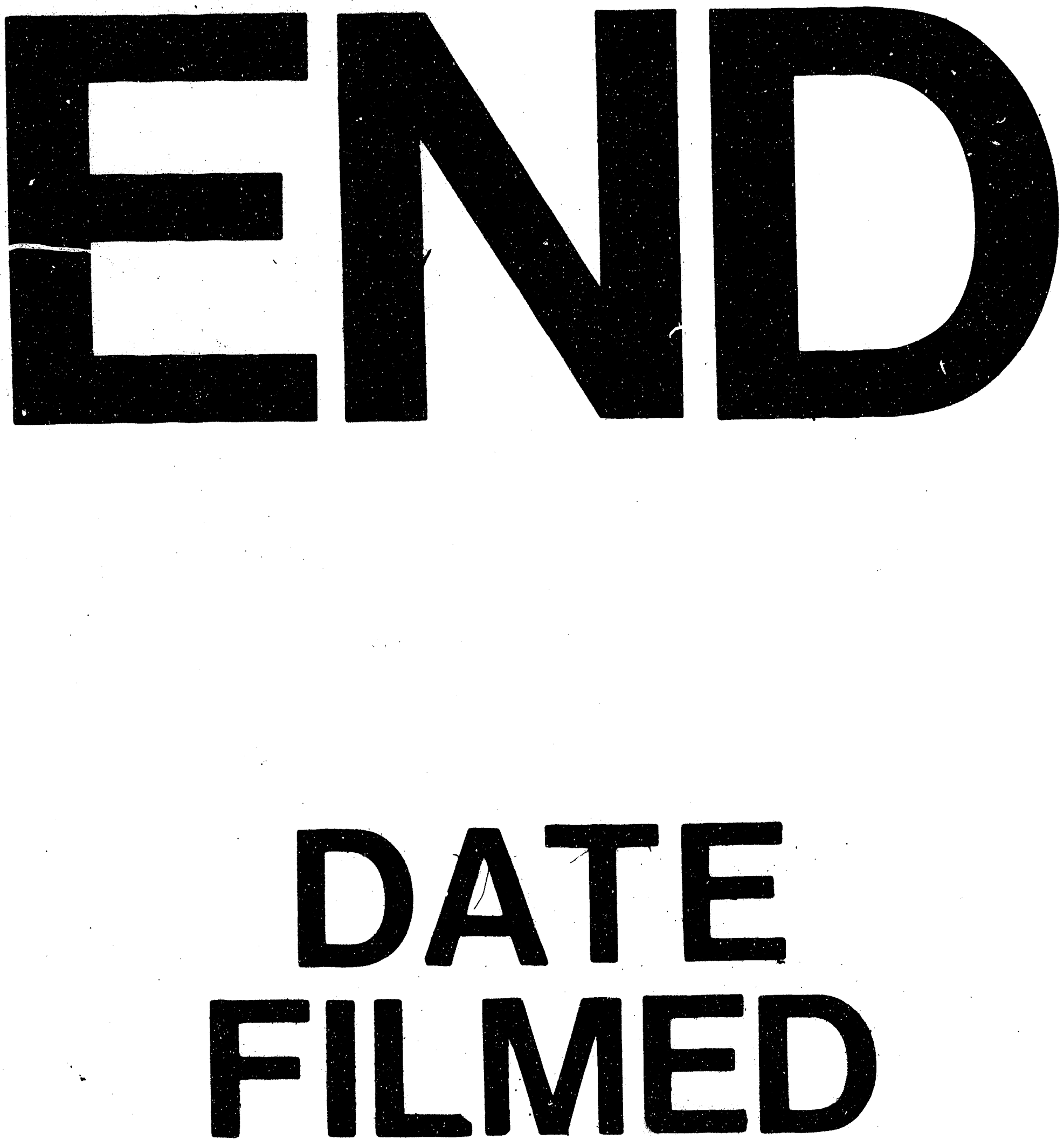

$\pm$

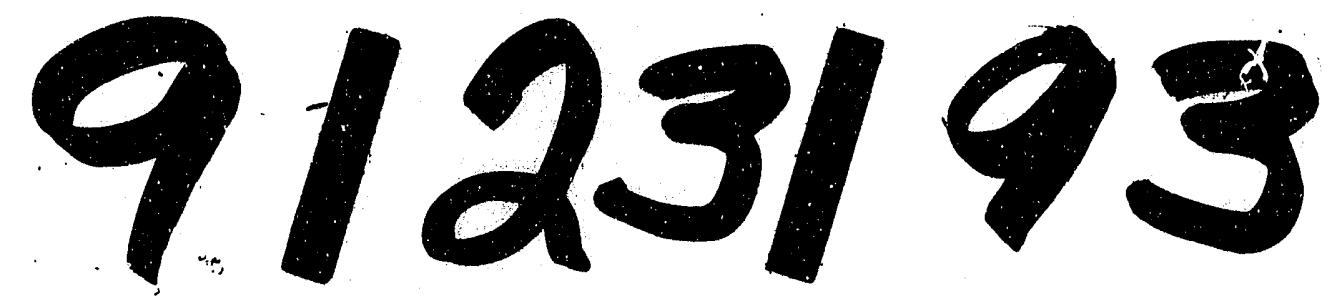


